\title{
AN UNCOUNTABLE FAMILY OF COPIES OF A NON-CHAINABLE TREE-LIKE CONTINUUM IN THE PLANE
}

\author{
L. C. HOEHN
}

(Communicated by Alexander N. Dranishnikov)

\begin{abstract}
A well-known theorem of R. L. Moore states that the plane does not contain an uncountable family of pairwise disjoint triods. In 1974, Ingram demonstrated that the same is not true for non-chainable tree-like continua. The continua in Ingram's family are not pairwise homeomorphic, making the example less applicable to the study of homogeneous continua in the plane. In this paper, we construct a non-chainable tree-like continuum $X$ such that the product of $X$ with the Cantor set can be embedded in the plane.
\end{abstract}

\section{INTRODUCTION}

By a continuum, we mean a compact connected metric space. A triod is a continuum $T$ having a subcontinuum $Z$ such that $T \backslash Z$ can be written as the union of three disjoint non-empty open sets. A continuum is tree-like if it is an inverse limit of trees, and chainable (or arc-like) if it is an inverse limit of arcs.

R. L. Moore proved in 1928 9] that the plane does not contain an uncountable family of pairwise disjoint triods. This has come to be a well-known and fundamental theorem with a wide variety of applications in topology and analysis in the plane.

If one considers only families of mutually homeomorphic compact spaces, stronger results are available. In particular, van Douwen 15 proved that if $X$ is any compactum and $\mathcal{C}$ is the Cantor set, then either $X \times \mathcal{C}$ can be embedded in the plane or there is no uncountable family of pairwise disjoint copies of $X$ in the plane (see [13] for a sharper dichotomy).

If $X$ is a triod, then $X$ fails the first alternative of van Douwen's theorem in a strong sense: if $S$ is any infinite compact space, then $X \times S$ cannot be embedded in the plane. In light of this observation, one may wonder what degree of tree "shape" $X$ must possess for a similar result to hold. Along these lines, Fugate asked (see [1, Problem 107]) whether $X \times S$ can be embedded in the plane if $X$ is a continuum which is neither chainable nor circle-like and $S$ is the convergent sequence $\{0\} \cup\left\{\frac{1}{n}: n=1,2, \ldots\right\}$.

In 1974, Ingram [5] (see also [6]) produced an example of an uncountable family of pairwise disjoint tree-like non-chainable continua in the plane, thus demonstrating that Moore's theorem cannot be generalized to non-chainable tree-like continua. However, the continua in Ingram's collection are not mutually homeomorphic.

Received by the editors October 11, 2011.

2010 Mathematics Subject Classification. Primary 54F15, 54F50.

Key words and phrases. Uncountable family, non-chainable, tree-like, continuum, plane. 
The topic of uncountable families of copies of a continuum in the plane has connections with the classical problem of classifying all homogeneous continua in the plane, which was first raised by Knaster and Kuratowski [8]. By results of Jones [7, Hagopian [3], and Oversteegen and Tymchatyn [11] and [12, if there is a non-degenerate homogeneous plane continuum other than the circle, the pseudoarc, and the circle of pseudo-arcs, then there would be one, $M$, which is hereditarily indecomposable, tree-like (in fact, with span zero), and which has a non-chainable proper subcontinuum $X$. Using autohomeomorphisms of $M$, one could then obtain a copy of $X$ in each composant of $M$; since $M$ is indecomposable, it has uncountably many composants, and they are pairwise disjoint.

Moreover, such a space $M$ (like any locally compact homogeneous Polish space) satisfies the so-called Effros property (see [2] and [14]): for any point $x \in M$ and any $\varepsilon>0$, there is a $\delta>0$ such that for any $y \in M$ within distance $\delta$ of $x$, there is an $\epsilon$-homeomorphism $h: M \rightarrow M$ such that $h(x)=y$. Using this, one can obtain copies of $X$ in $M$ which can be mapped to $X$ by arbitrarily small autohomeomorphisms of $M$. Thus, in particular, such a space $M$ would immediately provide a counterexample to Fugate's question.

Recently, the author constructed an example of a non-chainable continuum in the plane with span zero [4]. In this paper, we construct such a continuum $X$ and an embedding of $X \times \mathcal{C}$ in the plane, which gives a negative answer to Fugate's question. Moreover, we prove that for any $\varepsilon>0$ there is a $\delta>0$ such that if $p, q \in \mathcal{C}$ are at distance $<\delta$, then there is an $\varepsilon$-homeomorphism of the entire plane to itself which takes $X \times\{p\}$ to $X \times\{q\}$.

\section{Preliminaries}

We refer the reader to [10 for basic definitions concerning continua.

Denote by SEQ the set of finite sequences of zeros and ones, including the empty sequence $\emptyset$. If $s \in \mathrm{SEQ}, j \in\{0,1\}$, and $n \in \mathbb{N} \cup\{0\}$, then $s j$ is the sequence obtained by adding a single $j$ to the end of $s$, and $s\lceil n$ is the restriction of $s$ to $n$, i.e. the sequence consisting of the first $n$ elements of $s$. By $0^{n}$ (respectively $1^{n}$ ) we mean the sequence consisting of $n$ 0's (respectively $n 1$ 's).

We will identify the Cantor set $\mathcal{C}$ with the set of functions from $\mathbb{N} \cup\{0\}$ to $\{0,1\}$, with metric $d$ given by $d(y, z)=2^{-m}$, where $m$ is the smallest integer such that $y(m) \neq z(m)$. Given $y \in \mathcal{C}$ and $n \in \mathbb{N} \cup\{0\}$, denote by $y\lceil n$ the restriction of $y$ to $n$, which is an element of SEQ of length $n$.

We now recall some notation and terminology from [4. Let $\Gamma$ be the set of formal symbols $\{a, b, c\} \cup\left\{b_{t}: t \in[0,1]\right\}$. By a graph we mean an undirected connected graph without multiple edges joining the same pair of vertices and without any edge from a vertex to itself. If $G$ is a graph, $V(G)$ denotes the set of vertices. A simple triod is a space consisting of three arcs which have one common endpoint and are otherwise pairwise disjoint. All graphs considered in this paper will be simple triods, whose legs are divided into numerous edges. If $T$ is a simple triod and $p, q \in T$, then $[p, q]_{T}$ denotes the arc in $T$ with endpoints $p$ and $q$ (we may drop the subscript $T$ when it ought to be clear from the context).

A graph-word (in the alphabet $\Gamma$ ) is a pair $\rho=\left\langle G_{\rho}, w_{\rho}\right\rangle$, where $G_{\rho}$ is a graph, and $w_{\rho}: V\left(G_{\rho}\right) \rightarrow \Gamma$ is a function. 
For each positive integer $N$, denote by $\alpha_{N}, \beta_{N}, \gamma_{N}$ the following three words:

$$
\begin{aligned}
& (a b c)^{2 N+1} \prod_{i=0}^{2 N-1}\left[a b_{i / 2 N} c b_{i / 2 N} a(c b a)^{2 N-i-1} c b c(a b c)^{2 N-i-1}\right] a b_{1} c b_{1} a(c b a)^{2 N+1}, \\
& (a b c)^{2 N+1} \prod_{i=0}^{2 N-1}\left[a b_{i / 2 N} c b_{i / 2 N} a(c b a)^{2 N-i-1} c b a b c(a b c)^{2 N-i-1}\right] a b_{1} c b_{1} a(c b a)^{2 N+1} c b,
\end{aligned}
$$

ac.

Let $\left|\alpha_{N}\right|,\left|\beta_{N}\right|$ denote the lengths of the words $\alpha_{N}$ and $\beta_{N}$, respectively.

Define the graph-word $\rho_{N}$ as follows. Let $G_{\rho_{N}}$ be a simple triod, with vertex set $V\left(G_{\rho_{N}}\right)=\left\{o, p_{1}, \ldots, p_{\left|\alpha_{N}\right|-1}, q_{1}, \ldots, q_{\left|\beta_{N}\right|-1}, r\right\}$, where $o$ is the branch point of the triod, $p_{\left|\alpha_{N}\right|-1}, q_{\left|\beta_{N}\right|-1}, r$ are the endpoints of $G_{\rho_{N}}$, the points $p_{j}$ belong to the leg $\left[o, p_{\left|\alpha_{N}\right|-1}\right]$ with $p_{j} \in\left[o, p_{j+1}\right]$ for each $j$, and the points $q_{j}$ belong to the leg $\left[o, q_{\left|\beta_{N}\right|-1}\right]$ with $q_{j} \in\left[o, q_{j+1}\right]$ for each $j$. Put $p_{0}:=o$ and $q_{0}:=o$. Define $w_{\rho_{N}}$ : $V\left(G_{\rho_{N}}\right) \rightarrow \Gamma$ by $w_{\rho_{N}}\left(p_{j}\right):=\alpha_{N}(j), w_{\rho_{N}}\left(q_{j}\right):=\beta_{N}(j)$, and $w_{\rho_{N}}(r):=\gamma_{N}(1)=c$.

Definition. Given a simple triod $T$ with branch point $o$, a $\Gamma$-marking of $T$ is a function $\iota: \Gamma \rightarrow T$ such that $\iota(a), \iota(b), \iota(c)$ are the endpoints of $T$ and $\left\{\iota\left(b_{t}\right)\right.$ : $t \in[0,1]\} \subset[o, \iota(b)]$ are such that whenever $t<t^{\prime}$, we have $\iota\left(b_{t}\right) \in\left[o, \iota\left(b_{t^{\prime}}\right)\right]$ and $\operatorname{diam}\left(\left[\iota\left(b_{t}\right), \iota\left(b_{t^{\prime}}\right)\right]\right)=d\left(\iota\left(b_{t}\right), \iota\left(b_{t^{\prime}}\right)\right)=t^{\prime}-t$.

Definition. Let $\langle\Omega, d\rangle$ be a metric space, let $T \subseteq \Omega$ be a $\Gamma$-marked simple triod, let $G \subseteq \Omega$ be a graph, and let $\varepsilon>0$. Then $\rho=\langle G, w\rangle$ is a $\langle T, \varepsilon\rangle$-sketch of $G$ in $\Omega$ if there is a map $\widehat{w}: G \rightarrow T$ such that

(i) $\left.\widehat{w}\right|_{V(G)}=\iota \circ w$

(ii) for any adjacent $v_{1}, v_{2} \in V(G)$, we have that $\left.\widehat{w}\right|_{\left[v_{1}, v_{2}\right]_{G}}$ is a homeomorphism from $\left[v_{1}, v_{2}\right]_{G}$ to $\left[\iota\left(w\left(v_{1}\right)\right), \iota\left(w\left(v_{2}\right)\right)\right]_{T} ;$ and

(iii) $d(x, \widehat{w}(x))<\frac{\varepsilon}{2}$ for every $x \in G$.

In [4] it is proved that if a sequence of graphs $G_{\rho_{N_{n}}}$ is embedded in the plane so that $\rho_{N_{n+1}}$ is a $\left\langle G_{\rho_{N_{n}}}, \varepsilon_{n}\right\rangle$-sketch of $G_{\rho_{N_{n+1}}}$ for each $n$, where $\varepsilon_{n} \rightarrow 0$ fast enough, then the nested intersection of the $\varepsilon_{n}$-neighborhoods of these graphs is a non-chainable simple triod-like (in particular, tree-like) continuum. Moreover, if $N_{n} \rightarrow \infty$, then this intersection has span zero. Since the concept of span is not relevant for the present result, we will omit the definition.

\section{3. $(1+\varepsilon)$-BI-LIPSCHITZ MAPS AND $\varepsilon$-MAPS}

In this section we establish some basic results concerning $(1+\varepsilon)$-bi-Lipschitz maps and $\varepsilon$-maps of the plane.

Definition. Let $f: \mathbb{R}^{2} \rightarrow \mathbb{R}^{2}$ be a function, and let $\varepsilon>0$.

- $f$ is $(1+\varepsilon)$-bi-Lipschitz if $\frac{1}{1+\varepsilon} d\left(x_{1}, x_{2}\right) \leq d\left(f\left(x_{1}\right), f\left(x_{2}\right)\right) \leq(1+\varepsilon) d\left(x_{1}, x_{2}\right)$ for all $x_{1}, x_{2} \in \mathbb{R}^{2}$.

- $f$ is an $\varepsilon$-map if $d(x, f(x))<\varepsilon$ for all $x \in \mathbb{R}^{2}$.

Proposition 1. Let $\Phi_{1}: \mathbb{R}^{2} \rightarrow \mathbb{R}^{2}$ be $(1+\delta)$-bi-Lipschitz, and let $\Phi_{2}: \mathbb{R}^{2} \rightarrow \mathbb{R}^{2}$ be an $\varepsilon$-map. Then $\Phi_{1} \circ \Phi_{2} \circ \Phi_{1}^{-1}$ is an $(\epsilon \cdot(1+\delta))$-map. 
Proof. Given any $x \in \mathbb{R}^{2}$, we have

$$
\begin{aligned}
d\left(x, \Phi_{1} \circ \Phi_{2} \circ \Phi_{1}^{-1}(x)\right) & =d\left(\Phi_{1}\left(\Phi_{1}^{-1}(x)\right), \Phi_{1}\left(\Phi_{2}\left(\Phi_{1}^{-1}(x)\right)\right)\right) \\
& \leq(1+\delta) \cdot d\left(\Phi_{1}^{-1}(x), \Phi_{2}\left(\Phi_{1}^{-1}(x)\right)\right) \\
& \leq \varepsilon \cdot(1+\delta) .
\end{aligned}
$$

Lemma 2. Let $A_{1}, A_{2} \subset \mathbb{R}^{2}$ be straight line segments parameterized linearly by $h_{i}:[0,1] \rightarrow A_{i}(i=1,2)$ such that the straight line segments between points $h_{1}(t)$, $h_{2}(t)(t \in[0,1])$ foliate a quadrilateral $Q$ in $\mathbb{R}^{2}$. For any $\varepsilon>0$, there exists $\delta>0$ such that if $f:[0,1] \rightarrow[0,1]$ is a $(1+\delta)$-bi-Lipschitz $\delta$-map with $f(0)=0$ and $f(1)=1$, then the function $F: Q \rightarrow Q$ defined by

$$
F\left((1-s) h_{1}(t)+s h_{2}(t)\right)=(1-s) h_{1}(f(t))+s h_{2}(f(t)) \quad \text { for } s, t \in[0,1]
$$

is $a(1+\varepsilon)$-bi-Lipschitz $\varepsilon$-map.

Proof. Given $s, t \in[0,1]$, let $p_{s, t}=(1-s) h_{1}(t)+s h_{2}(t)$, and let $\alpha_{s, t}$ be the angle, $0 \leq \alpha_{s, t} \leq \frac{\pi}{2}$, between the line containing $p_{0, t}$ and $p_{1, t}$ and the line containing $p_{s, 0}$ and $p_{s, 1}$. Observe that there exists some $0<\alpha_{0} \leq \frac{\pi}{2}$, dictated by the geometry of $Q$, such that $\alpha_{0} \leq \alpha_{s, t} \leq \frac{\pi}{2}$ for all $s, t \in[0,1]$.

It is straightforward to see that there exists $\delta_{0}>0$ small enough so that the following hold whenever $f$ is a $\delta_{0}$-map:

(i) $F$ is an $\varepsilon$-map.

(ii) If $s, t \in[0,1]$, then $1-\frac{\varepsilon}{2}<\frac{\left|\cos \left(\alpha_{s, t}\right)\right|}{\left|\cos \left(\alpha_{s, f(t)}\right)\right|}<\left(1-\frac{\varepsilon}{2}\right)^{-1}$.

(iii) If $s_{1}, s_{2}, t \in[0,1]$, then $(\sqrt{1+\varepsilon})^{-1}<\frac{d\left(p_{s_{1}, t}, p_{s_{2}, t}\right)}{d\left(p_{s_{1}, f(t)}, p_{s_{2}, f(t)}\right.}<\sqrt{1+\varepsilon}$.

Moreover, there exists $\delta_{1}>0$ small enough so that the following holds whenever $f$ is $\left(1+\delta_{1}\right)$-bi-Lipschitz:

(iv) If $s, t_{1}, t_{2} \in[0,1]$, then $(\sqrt{1+\varepsilon})^{-1}<\frac{d\left(p_{\left.s, t_{1}, p_{s, t_{2}}\right)}\right.}{d\left(p_{s, f\left(t_{1}\right)}, p_{s, f\left(t_{2}\right)}\right)}<\sqrt{1+\varepsilon}$.

Let $\delta=\min \left\{\delta_{0}, \delta_{1}\right\}$, and suppose $f$ is a $(1+\delta)$-bi-Lipschitz $\delta$-map. Given any $s_{1}, s_{2}, t_{1}, t_{2} \in[0,1]$, let

$$
\begin{array}{ll}
A=d\left(p_{s_{1}, t_{1}}, p_{s_{2}, t_{1}}\right), & A^{\prime}=d\left(p_{s_{1}, f\left(t_{1}\right)}, p_{s_{2}, f\left(t_{1}\right)}\right), \\
B=d\left(p_{s_{2}, t_{1}}, p_{s_{2}, t_{2}}\right), & B^{\prime}=d\left(p_{s_{2}, f\left(t_{1}\right)}, p_{s_{2}, f\left(t_{2}\right)}\right), \\
C=d\left(p_{s_{1}, t_{1}}, p_{s_{2}, t_{2}}\right), & C^{\prime}=d\left(p_{s_{1}, f\left(t_{1}\right)}, p_{s_{2}, f\left(t_{2}\right)}\right), \\
\alpha=\alpha_{s_{2}, t_{1}}, & \alpha^{\prime}=\alpha_{s_{2}, f\left(t_{1}\right)} .
\end{array}
$$

Using the law of cosines and properties (ii), (iii), and (iv), we have

$$
\begin{aligned}
\left(C^{\prime}\right)^{2} & =\left(A^{\prime}\right)^{2}+\left(B^{\prime}\right)^{2}-2 A^{\prime} B^{\prime} \cos \left(\alpha^{\prime}\right) \\
& \leq(1+\varepsilon) A^{2}+(1+\varepsilon) B^{2}-2(1+\varepsilon) A B \cos \left(\alpha^{\prime}\right) \\
& \leq(1+\varepsilon) A^{2}+(1+\varepsilon) B^{2}-2(1+\varepsilon)\left(1-\frac{\varepsilon}{2}\right) A B \cos (\alpha) \\
& \leq(1+\varepsilon) A^{2}+(1+\varepsilon) B^{2}-2(1+\varepsilon) A B \cos (\alpha)+(1+\varepsilon) \varepsilon C^{2} \\
& =(1+\varepsilon) C^{2}+(1+\varepsilon) \varepsilon C^{2} \\
& =(1+\varepsilon)^{2} C^{2} .
\end{aligned}
$$

Therefore $d\left(F\left(p_{s_{1}, t_{1}}\right), F\left(p_{s_{2}, t_{2}}\right)\right)=C^{\prime} \leq(1+\varepsilon) C=(1+\varepsilon) d\left(p_{s_{1}, t_{1}}, p_{s_{2}, t_{2}}\right)$. Likewise we can obtain $d\left(p_{s_{1}, t_{1}}, p_{s_{2}, t_{2}}\right) \leq(1+\varepsilon) d\left(F\left(p_{s_{1}, t_{1}}\right), F\left(p_{s_{2}, t_{2}}\right)\right)$. Thus $F$ is $(1+\varepsilon)$ bi-Lipschitz. 
See Figure 5 below for an illustration of a scenario satisfying the hypotheses of the next lemma.

Lemma 3. Let $A_{1}, \ldots, A_{n} \subset \mathbb{R}^{2}$ be straight line segments parameterized linearly by $h_{i}:[0,1] \rightarrow A_{i}(i=1, \ldots, n)$, and let $A_{n+1}=A_{1}, h_{i+1}=h_{1}$. Suppose that the straight line segments between points $h_{i}(t), h_{i+1}(t)(t \in[0,1])$ foliate a quadrilateral $Q_{i}$ in $\mathbb{R}^{2}$, for $i=1, \ldots, n$. Assume that $Q_{i} \cap Q_{i+1}=A_{i+1}$ for each $i=1, \ldots, n-1$, that $Q_{n} \cap Q_{1}=A_{1}$, and that $Q_{i} \cap Q_{j}=\emptyset$ for all other pairs $i, j$. For any $\varepsilon>0$, there exists $\delta>0$ such that if $f:[0,1] \rightarrow[0,1]$ is a $(1+\delta)$-bi-Lipschitz $\delta$-map with $f(0)=0$ and $f(1)=1$, then the function $\Phi: \mathbb{R}^{2} \rightarrow \mathbb{R}^{2}$ defined like the function $F$ in Lemma 2 on each $Q_{i}$, and with $\Phi=\mathrm{id}$ on $\mathbb{R}^{2} \backslash \bigcup_{i=1}^{n} Q_{i}$, is a $(1+\varepsilon)$-bi-Lipschitz $\varepsilon$-map.

Proof. Given $i \in\{1, \ldots, n\}$ and $s, t \in[0,1]$, let $p_{s, t}^{i}=(1-s) h_{i}(t)+s h_{i+1}(t)$. Let $x, y \in \mathbb{R}^{2}$ be given, and suppose $x=p_{s_{1}, t_{1}}^{i} \in Q_{i}$. Note that for some $j$, we can write $y=\left(1-s_{2}\right) h_{j}\left(t_{2}\right)+s_{2} h_{j+1}\left(t_{2}\right)$ for some $t_{2} \in[0,1]$ and $s_{2} \in \mathbb{R}$, even if $y \notin Q_{j}$ (i.e. if $\left.s_{2} \notin[0,1]\right)$. Then $x$ and $y$ can be joined by a piecewise straight line segment path with $k \leq n$ intermediate vertices $p_{0, t_{1}}^{i+1}, p_{0, t_{1}}^{i+2}, \ldots, p_{0, t_{1}}^{i+k}, p_{s_{1}, t_{1}}^{i+k}$ (here the superscripts are taken modulo $n$, and $i+k \equiv j \bmod n)$. As in the proof of Lemma 2, we can choose $\delta>0$ small enough so that the lengths of these segments and the angles between them may change only very slightly under $\Phi$. The remainder of the argument proceeds similarly to the proof of Lemma 2

\section{UnCountably many COPIES OF $X$ IN the Plane}

We will need the following strengthening of Proposition 1 of [4]. By piecewiselinear we mean composed of finitely many straight line segments.

Proposition 4. Suppose $T \subset \mathbb{R}^{2}$ is a piecewise-linear simple triod and $\iota: \Gamma \rightarrow T$ is a $\Gamma$-marking. For any positive integer $N_{0}$ and any $\varepsilon>0$, there is an integer $N \geq N_{0}$, a piecewise-linear embedding $G$ of the simple triod graph $G_{\rho_{N}}$ in $\mathbb{R}^{2}$, and a map $\Phi: \mathbb{R}^{2} \rightarrow \mathbb{R}^{2}$ such that:

(i) $\rho_{N}$ is a $\langle T, \varepsilon\rangle$-sketch of $G$ in $\mathbb{R}^{2}$;

(ii) $\left[q_{\left|\beta_{N}\right|-2}, q_{\left|\beta_{N}\right|-1}\right]_{G}=[\iota(c), \iota(b)]_{T}$;

(iii) $\Phi$ is a $(1+\varepsilon)$-bi-Lipschitz $\varepsilon$-map;

(iv) $G \cap \Phi(G)=\emptyset$.

Proof. For simplicity, as in [4, we argue only the case $T=T_{0}=\{(x, 0): x \in$ $[-1,1]\} \cup\{(0, y): y \in[0,2]\}$, and with the $\Gamma$-marking $\iota$ given by

$$
\begin{aligned}
\iota(a) & =(-1,0), \\
\iota(b) & =(0,2), \\
\iota(c) & =(1,0), \\
\iota\left(b_{t}\right) & =(0,1+t) \quad \text { for } t \in[0,1] .
\end{aligned}
$$

The general case can be treated similarly.

We recall the procedure described in [4] for constructing an embedding $G$ of $G_{\rho_{N}}$ near $T$ such that $\rho_{N}$ is a $\langle T, \varepsilon\rangle$-sketch of $G$ :

Step 1 . In a small neighborhood in the plane of the ray $(-\infty, 1]$ in the real line, we embed the simple triod graph $G_{\rho_{N}}$ as depicted in Figure 1 (illustrated for the case $N=1$; see also e.g. the black triod in Figure 4 for the case $N=2$ ). 


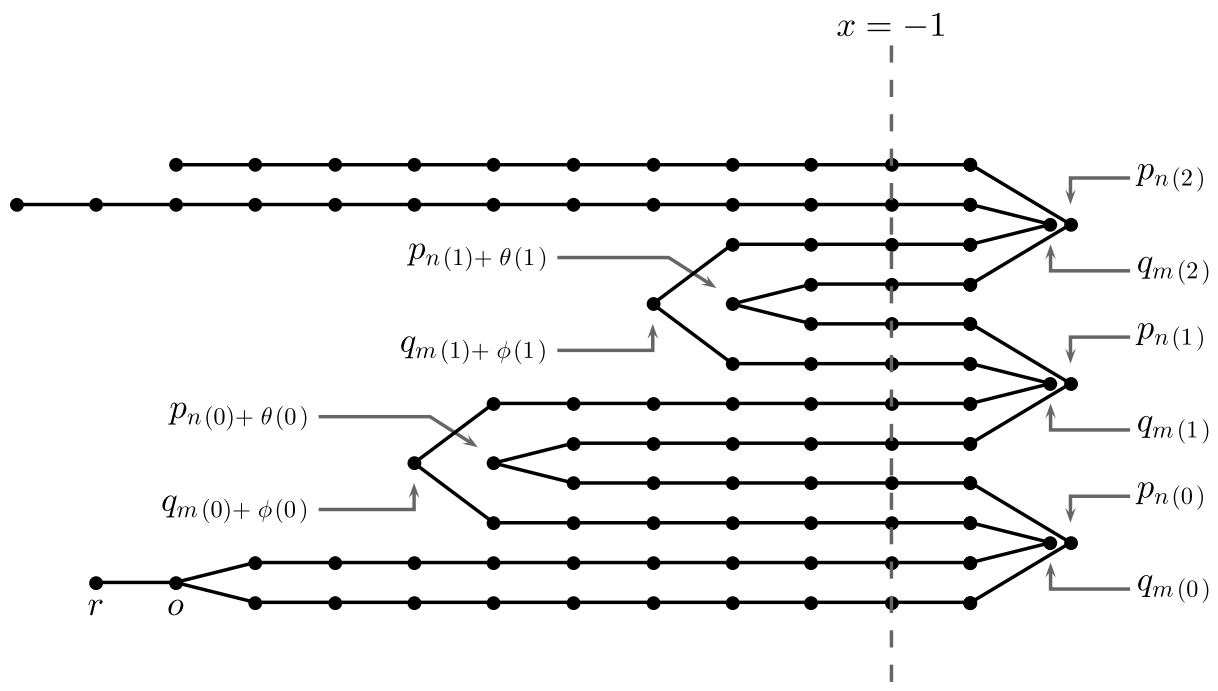

FiguRE 1. The first intermediate stage for the embedding of $G_{\rho_{1}}$ in $\mathbb{R}^{2}$. The labelled points are significant for the combinatorial arguments in [4].

Step 2. Consider the $8 N+4$ arcs to the right of $x=-1$ (which are joined in pairs at the right to form $4 N+2$ "V's"). These appear in $2 N+1$ quadruples (or $2 N+1$ pairs of "V's"). We take the $i$-th quadruple and pull the center part of each arc up, so as to shape it along the path $\left[\iota(a), \iota\left(b_{i / 2 N}\right)\right]_{T} \cup\left[\iota\left(b_{i / 2 N}\right), \iota(c)\right]_{T}$, as depicted in Figure 2 (illustrated for the case $N=1$ ).

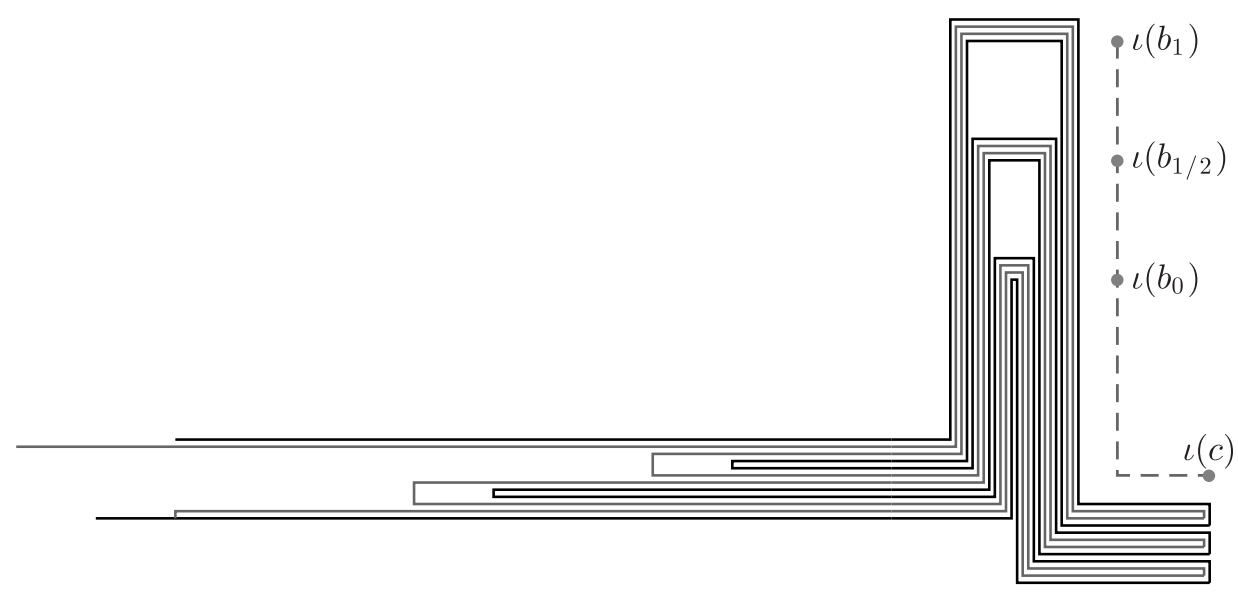

FIgURE 2. The second intermediate stage for the embedding of $G_{\rho_{1}}$ in $\mathbb{R}^{2}$. 
(a) Before wrapping

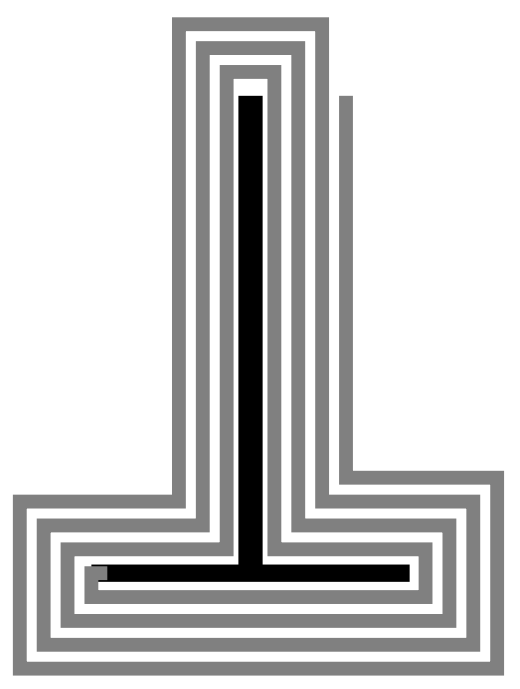

(b) After wrapping

FiguRE 3. Wrapping the strip counterclockwise around the simple triod to obtain the embedding of $G_{\rho_{N}}$ in $\mathbb{R}^{2}$.

Step 3. Take the bundle of arcs extending to the left of $x=-1$ and stretch and wind it counterclockwise around the outside of the section in Figure 2 to the right of $x=-1$. Figure 3 depicts roughly how this wrapping looks.

The substance of this proposition is summarized in Figure 4, which indicates how we can manage Step 1 above with two disjoint copies of $G_{\rho_{N}}$ (illustrated for the case $N=2$ ), then carry out Steps 2 and 3 with both copies simultaneously, obtaining two disjoint embeddings which can be moved to one another by a small map of the plane which is nearly an isometry. The remainder of the proof describes how to realize this precisely.

In the $\frac{\varepsilon}{8}$-neighborhood of $T$, we place a ring of quadrilaterals $Q_{1}, \ldots, Q_{6}$ as depicted in Figure 5, with arcs $A_{i}$ parameterized linearly by $h_{i}:[0,1] \rightarrow A_{i}$, where $h_{i}(0)$ is the endpoint of $A_{i}$ which is accessible from the bounded component of 


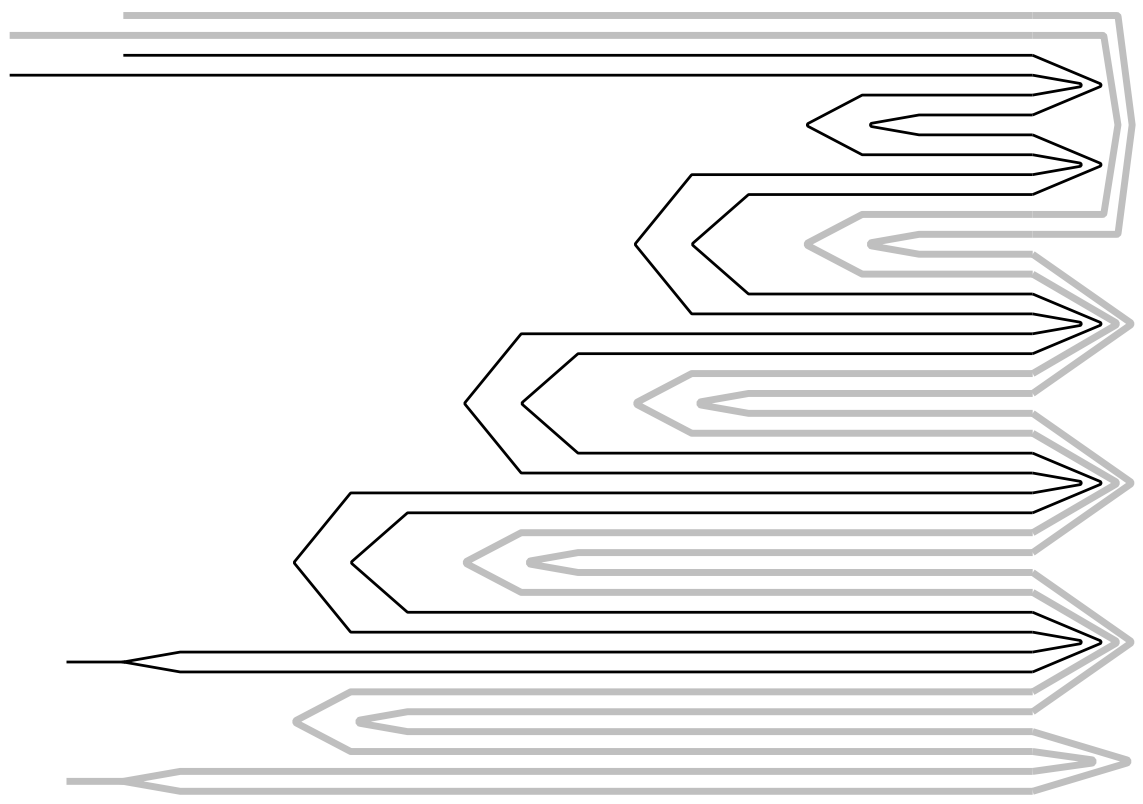

Figure 4. Two disjoint copies of the simple triod in the plane.

$\mathbb{R}^{2} \backslash \bigcup_{i=1}^{6} Q_{i}$. Place the $\operatorname{arcs} A_{1}, A_{2}, A_{3}$ so that $\iota(b) \in A_{1},(0,0) \in A_{2}$, and $\iota(c) \in A_{3}$. We will define an embedding $G$ of $G_{\rho_{N}}$ inside the union of these quadrilaterals.

Let $\delta>0$ be the number guaranteed by Lemma 3 for $\varepsilon$, and let $N \geq N_{0}$ be large enough so that $\frac{1}{N}<\delta$.

For $t \in\left\{0, \frac{1}{2 N}, \frac{2}{2 N}, \ldots, 1\right\}$, let $b_{t}^{\prime} \in A_{1}$ be such that $b_{t}^{\prime}$ is within distance $\frac{\varepsilon}{4}$ of $\iota\left(b_{t}\right)$, the points $h_{1}^{-1}\left(b_{t}^{\prime}\right)$ are evenly spaced in the interior of $[0,1]$, and $h_{1}^{-1}\left(b_{t}^{\prime}\right)<h_{1}^{-1}(\iota(b))$ for each $t$.

Let $f_{0}:[0,1] \rightarrow[0,1]$ be the map depicted in Figure 6, where it is understood that the slope of the graph of $f$ is always between $(1+\delta)^{-1}$ and $1+\delta$, so that $f_{0}$ is $(1+\delta)$-bi-Lipschitz. Note that $f_{0}$ is a $\frac{1}{2 N+1}$-map.

Let $f$ be the map built out of infinitely many copies of $f_{0}$ and a final section $R$ on which $f(s)>s$, as depicted in Figure 7 where the first (largest) copy of $f_{0}$ is properly scaled and positioned so that the marked points in this copy of $f_{0}$ in Figure 7 are precisely the points $h_{1}^{-1}\left(b_{t}^{\prime}\right)$ for $t \in\left\{0, \frac{1}{2 N}, \frac{2}{2 N}, \ldots, 1\right\}$. We also assume that $h_{1}^{-1}(\iota(b)), h_{2}^{-1}((0,0)), h_{3}^{-1}(\iota(c))$ each belong to the section $R$. Note that $f$ is a $(1+\delta)$-bi-Lipschitz $\delta$-map since $f_{0}$ is. Let $\Phi$ be defined as in Lemma 3 so that $\Phi$ is a $(1+\varepsilon)$-bi-Lipschitz $\varepsilon$-map.

In a small neighborhood of each point $h_{1}^{-1}\left(b_{i / 2 N}^{\prime}\right), 0 \leq i \leq 2 N-1$, choose four points $a_{1}^{i}, a_{2}^{i}, a_{3}^{i}, a_{4}^{i}$, so that for each $i<2 N-1$,

$$
f\left(a_{1}^{i+1}\right)<f\left(a_{2}^{i+1}\right)<a_{1}^{i}<a_{2}^{i}<a_{3}^{i}<a_{4}^{i}<f\left(a_{3}^{i+1}\right)<f\left(a_{4}^{i+1}\right)
$$

and $h_{1}\left(a_{j}^{i}\right)$ and $h_{1}\left(f\left(a_{j}^{i+1}\right)\right)$ are within $\frac{\varepsilon}{4}$ of $b_{i / 2 N}^{\prime}$, for each $j$. Moreover, in a small neighborhood of the point $h_{1}^{-1}\left(b_{1}^{\prime}\right)$, choose two points $a_{1}^{2 N}<a_{2}^{2 N}$ such that

$$
f\left(a_{1}^{2 N}\right)<f\left(a_{2}^{2 N}\right)<a_{1}^{2 N-1}
$$




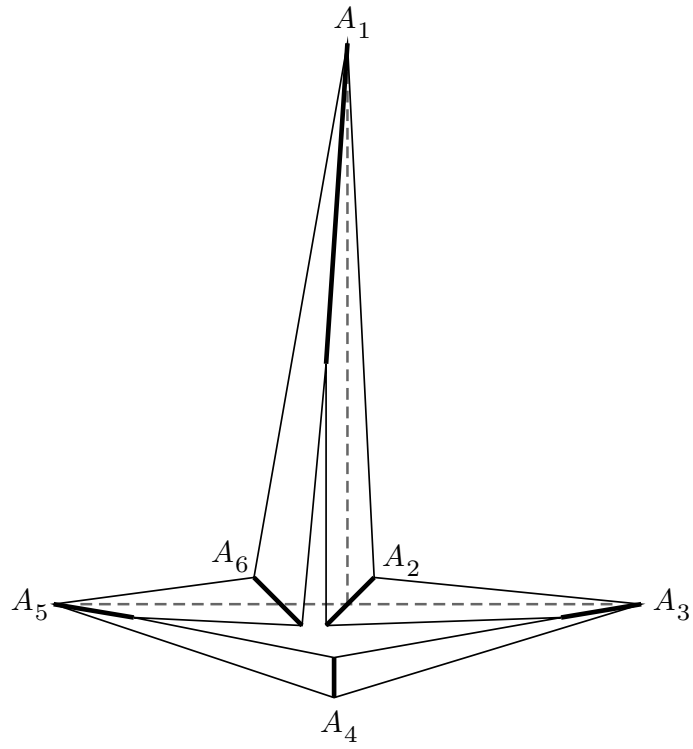

Figure 5. A ring of quadrilaterals in a small neighborhood of $T$ ( $T$ is shown in dashed lines). The asymmetry in the quadrilaterals is present in order to accomodate the requirement that $\left[q_{\left|\beta_{N}\right|-2}, q_{\left|\beta_{N}\right|-1}\right]_{G}=[\iota(c), \iota(b)]_{T}$.

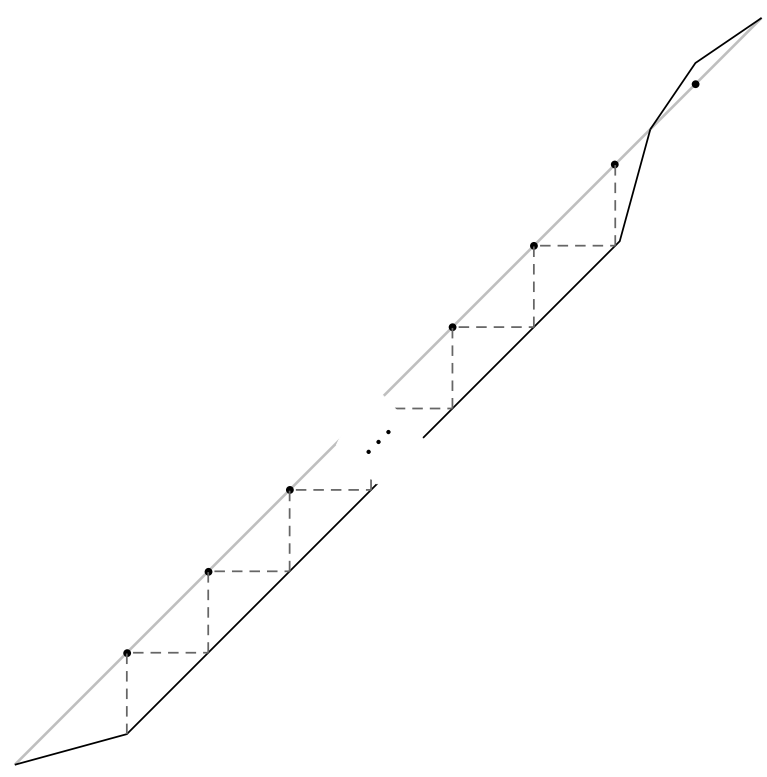

Figure 6. The function $f_{0}:[0,1] \rightarrow[0,1]$. 


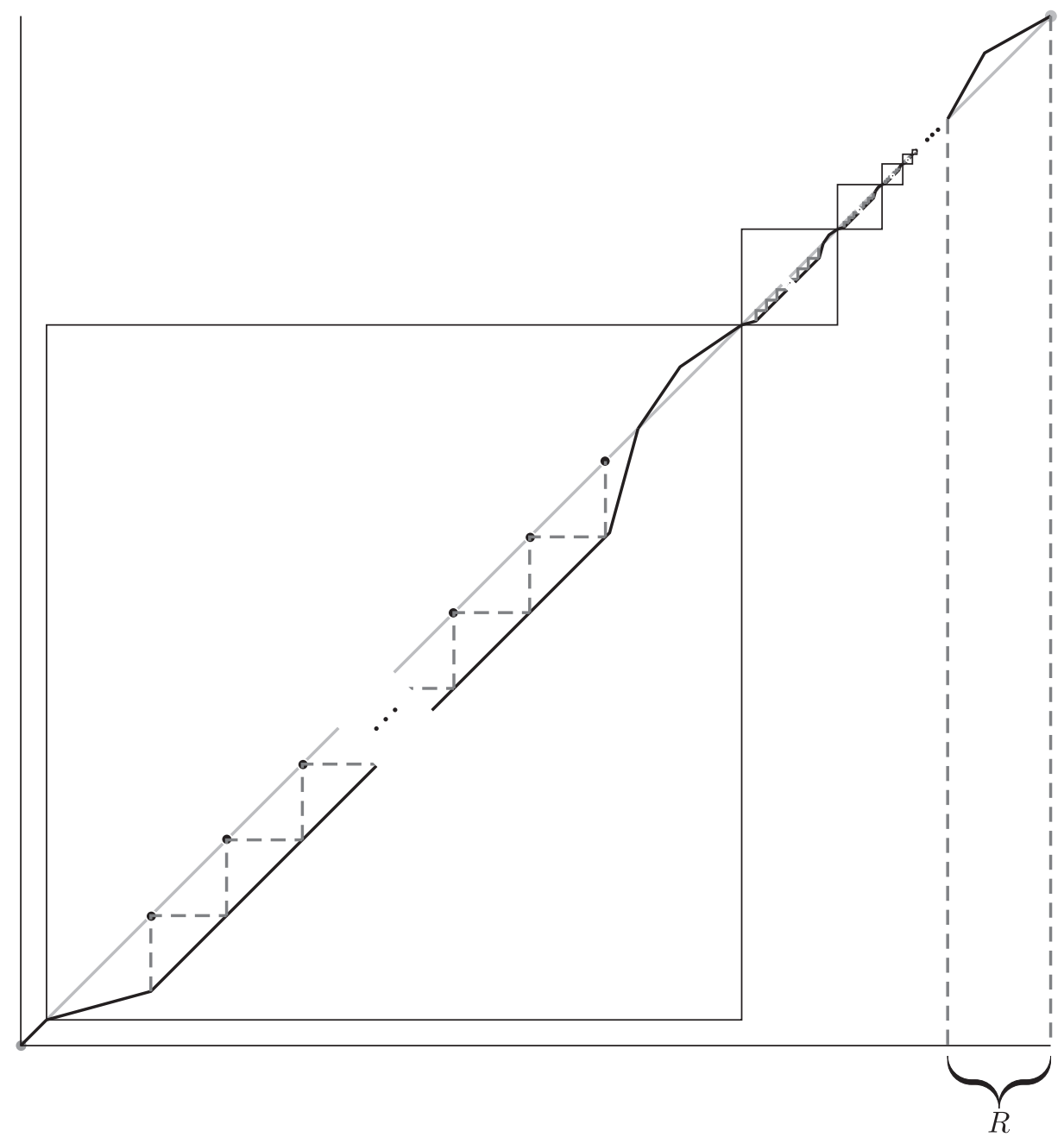

Figure 7 . The function $f:[0,1] \rightarrow[0,1]$, built out of countably many copies of $f_{0}$, and an increasing map $f: R \rightarrow R$.

and choose two points $a_{3}^{2 N}<a_{4}^{2 N}$ beyond the interior fixed point of the first copy of $f_{0}$ in $f$ so that

$$
a_{4}^{2 N}<f\left(a_{3}^{2 N}\right)<f\left(a_{4}^{2 N}\right)
$$

Between the points $h_{i}\left(a_{j}^{i}\right)$ we draw straight line segments as depicted in Figure 8 (illustrated for the case $N=1$ ) to accomplish Step 2 above. We then wrap the remaining arcs as per Step 3 around what is shown in Figure 8, using similar quadruples of points near the marked points from Figure 6 in the other copies of $f_{0}$ in $f$, making sure to thread the arc $\left[q_{\left|\beta_{N}\right|-2}, q_{\left|\beta_{N}\right|-1}\right]_{G}$ through the section $h_{i}(R)$ in $A_{i}(i=1,2,3)$ appropriately so as to satisfy property (ii). The embedding $G$ obtained this way is such that $\rho_{N}$ is a $\left\langle T_{0}, \varepsilon\right\rangle$-sketch of $G$. Moreover, $G$ is moved off itself by $\Phi$ in exactly the way indicated in Figure 4 and therefore satisfies property (v). 


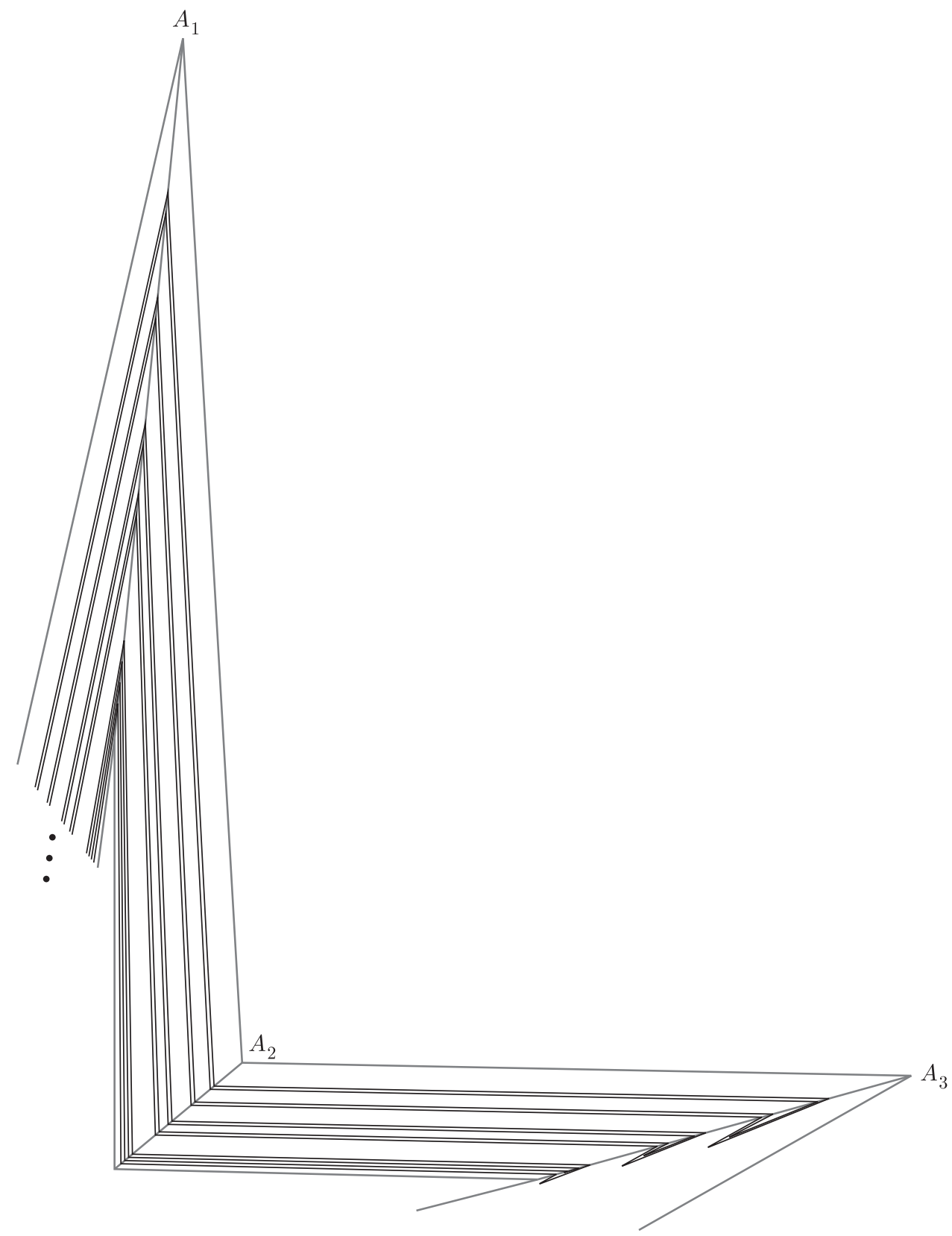

Figure 8. Performing Step 2 of the construction inside the quadrilaterals $Q_{1}, \ldots, Q_{6}$.

Using Proposition 4, we now construct the desired embedding of $X \times \mathcal{C}$ in $\mathbb{R}^{2}$. Fix a sequence of positive real numbers $\left\langle a_{n}\right\rangle_{n=0}^{\infty}$ such that $\sum_{n=m}^{\infty} a_{n} \leq 2^{-m}$ for each $m \geq 0$, and $\prod_{n=0}^{\infty}\left(1+a_{n}\right)^{2} \leq 2$. 
We will construct, by recursion, numbers $\varepsilon_{n}>0$, simple triods $T_{s} \subset \mathbb{R}^{2}, \Gamma$ markings $\iota_{n}$ of $T_{0^{n}}$, and maps $\Phi_{s, t}$ of the plane such that $\Phi_{s, t}\left(T_{s}\right)=T_{t}$, for $n \geq 0$ and $s, t \in \mathrm{SEQ}$ of length $n$.

Let $T_{\emptyset}=\{(x, 0): x \in[-1,1]\} \cup\{(0, y): y \in[0,2]\}, \Phi_{\emptyset, \emptyset}=\mathrm{id}$, and $\varepsilon_{0}=a_{0}$. Give $T_{\emptyset}$ the $\Gamma$-marking $\iota_{0}$ defined as follows:

$$
\begin{aligned}
\iota_{0}(a) & =(-1,0), \\
\iota_{0}(b) & =(0,2), \\
\iota_{0}(c) & =(1,0), \\
\iota_{0}\left(b_{t}\right) & =(0,1+t) \quad \text { for } t \in[0,1] .
\end{aligned}
$$

Suppose $\varepsilon_{n}, T_{s}, \iota_{n}$, and $\Phi_{s, t}$ have been defined for all $s, t \in \mathrm{SEQ}$ of length $n$. Let $0<\varepsilon_{n}^{\prime}<\varepsilon_{n}$ be small enough so that

$$
\overline{\Phi_{0^{n}, s}\left(\left(T_{0^{n}}\right)_{\varepsilon_{n}^{\prime}}\right)} \subset\left(T_{s}\right)_{\varepsilon_{n}}
$$

for all $s \in \mathrm{SEQ}$ of length $n$.

Apply Proposition 4 to obtain an integer $N_{n+1}>N_{n}$, disjoint simple triods $T_{0^{n} 0}, T_{0^{n} 1}$, and a $\left(1+\varepsilon_{n}^{\prime}\right)$-bi-Lipschitz $\frac{\varepsilon_{n}^{\prime}}{2}$-map $\Phi_{0^{n} 0,0^{n} 1}: \mathbb{R}^{2} \rightarrow \mathbb{R}^{2}$ such that $\Phi_{0^{n} 0,0^{n} 1}\left(T_{0^{n} 0}\right)=T_{0^{n} 1}$ and $\rho_{N_{n+1}}$ is a $\left\langle T_{0^{n}}, \varepsilon_{n}^{\prime}\right\rangle$-sketch of $T_{0^{n} 0}$. Moreover assume $\left[q_{\left|\beta_{N}\right|-2}, q_{\left|\beta_{N}\right|-1}\right]_{T_{0^{n}} 0}=\left[\iota_{n}(c), \iota_{n}(b)\right]_{T_{0^{n}}}$, where here the points $p_{j}, q_{j}$, and $r$ refer to the vertices of $T_{0^{n} 0}$ considered as an embedding of the graph $G_{\rho_{N_{n+1}}}$.

As in [4, we define a $\Gamma$-marking $\iota_{n+1}: \Gamma \rightarrow T_{0^{n} 0}$ on $T_{0^{n} 0}$ as follows: define $\iota_{n+1}(a):=p_{\left|\alpha_{N}\right|-1}, \iota_{n+1}(b):=q_{\left|\beta_{N}\right|-1}=\iota_{n}(b), \iota_{n+1}(c):=r$, and for each $t \in[0,1]$ put $\iota_{n+1}\left(b_{t}\right):=\iota_{n}\left(b_{t}\right) \in\left[q_{\left|\beta_{N}\right|-2}, q_{\left|\beta_{N}\right|-1}\right]_{T_{0^{n}}}=\left[\iota_{n}(c), \iota_{n}(b)\right]_{T_{0^{n}}}$.

Put $\Phi_{0^{n+1}, 0^{n+1}}=$ id. For $s \in \mathrm{SEQ}$ of length $n+1$, define

$$
\Phi_{0^{n+1}, s}=\Phi_{0^{n}, s \uparrow(n+1)} \circ \Phi_{0^{n} 0,0^{n} s(n)} \quad \text { and } \quad T_{s}=\Phi_{0^{n+1}, s}\left(T_{0^{n+1}}\right) .
$$

Observe that $\Phi_{0^{n} 0,0^{n} s(n)}\left(T_{0^{n+1}}\right) \subset\left(T_{0^{n}}\right)_{\varepsilon_{n}^{\prime}}$, so by (1) we have $T_{s} \subset\left(T_{s \uparrow(n+1)}\right)_{\varepsilon_{n}}$.

For $s, t \in \mathrm{SEQ}$ of length $n+1$, define $\Phi_{s, t}=\Phi_{0^{n+1}, t} \circ \Phi_{0^{n+1}, s}^{-1}$.

Observe that

$$
\Phi_{0^{n+1}, s}=\Phi_{0, s(0)} \circ \Phi_{0^{2}, 0 s(1)} \circ \cdots \circ \Phi_{0^{n+1}, 0^{n} s(n)},
$$

and so $\Phi_{0^{n+1}, s}$ is a $\prod_{i=0}^{n+1}\left(1+\varepsilon_{i}\right)$-bi-Lipschitz map. It follows that $\Phi_{s, t}$ is $\prod_{i=0}^{n+1}(1+$ $\left.\varepsilon_{i}\right)^{2}$-bi-Lipschitz; in particular,

$$
\Phi_{s, t} \text { is 2-bi-Lipschitz. }
$$

Let $\varepsilon_{n+1}<a_{n+1}$ be small enough so that for each $s, t \in \mathrm{SEQ}$ of length $n+1$, we have $\overline{\left(T_{s}\right)_{\varepsilon_{n+1}}} \subset\left(T_{s \uparrow(n+1)}\right)_{\varepsilon_{n}}$ and $\overline{\left(T_{s}\right)_{\varepsilon_{n+1}}} \cap \overline{\left(T_{t}\right)_{\varepsilon_{n+1}}}=\emptyset$.

For any $y \in \mathcal{C}$, define $X_{y}=\bigcap_{n=0}^{\infty} \overline{\left(T_{y\lceil n}\right)_{\varepsilon_{n}}}$. Observe that if $y, z \in \mathcal{C}$ with $y \neq z$, then $X_{y} \cap X_{z}=\emptyset$, since $\overline{\left(T_{y\lceil n}\right)_{\varepsilon_{n}}} \cap \overline{\left(T_{z \nmid n}\right)_{\varepsilon_{n}}}=\emptyset$ for any $n$ such that $y(n) \neq z(n)$. It was proved in [4] that $X=X_{\overline{0}}$ is a non-chainable tree-like continuum (having span zero).

Fix $y, z \in \mathcal{C}$ with $y \neq z$, and let $m$ be maximal such that $y\lceil m=z\lceil m$. Note that $\Phi_{y\lceil m, z\lceil m}=\mathrm{id}$. Given $n \geq m$, suppose that $\Phi_{y\lceil n, z \nmid n}$ is a $\left(\sum_{i=m}^{n-1} \varepsilon_{i}\right)$-map. Observe that

$$
\Phi_{y \uparrow(n+1), z \uparrow(n+1)}=\Phi_{0^{n}, z\lceil n} \circ \Phi_{0^{n} y(n), 0^{n} z(n)} \circ \Phi_{0^{n}, z\lceil n}^{-1} \circ \Phi_{y\lceil n, z\lceil n} .
$$


Since $\Phi_{0^{n} y(n), 0^{n} z(n)}$ is an $\frac{\varepsilon_{n}}{2}$-map and $\Phi_{0^{n}, z \nmid n}$ is 2-bi-Lipschitz, we have by Proposition 1 that

$$
\Phi_{0^{n}, z\lceil n} \circ \Phi_{0^{n} y(n), 0^{n} z(n)} \circ \Phi_{0^{n}, z \nmid n}^{-1} \text { is an } \varepsilon_{n^{-}} \text {map. }
$$

It follows that $\Phi_{y \uparrow(n+1), z \uparrow(n+1)}$ is a $\left(\sum_{i=m}^{n} \varepsilon_{i}\right)$-map. By induction, this establishes that

$$
\Phi_{y\lceil n, z \nmid n} \text { is a }\left(\sum_{i=m}^{n-1} \varepsilon_{i}\right) \text {-map, for each } n \geq m .
$$

Now define $\Phi_{y, z}=\lim _{n \rightarrow \infty} \Phi_{y \nmid n, z \nmid n}$ (pointwise). This is well defined by (3) and (4). By (21), we have that $\Phi_{y, z}$ is 2-bi-Lipschitz; in particular, it is a homeomorphism. Note that $\Phi_{y, z}\left(X_{y}\right)=X_{z}$ since $\Phi_{y \nmid n, z \nmid n}\left(T_{y \nmid n}\right)=T_{z \nmid n}$ for each $n$. Furthermore, by (5), we have that $\Phi_{y, z}$ is a $\left(\sum_{i=m}^{\infty} \varepsilon_{i}\right)$-map; in particular, it is a $2^{-m}$-map.

\section{Questions}

The construction presented in this paper has the property that every proper subcontinuum of the space $X$ is an arc; hence, in particular, it is far from being hereditarily indecomposable. Moreover, as shown in [4], $X$ has span zero. It is unknown whether these properties are necessary:

Question 1. Is there a hereditarily indecomposable non-chainable tree-like continuum $X$ such that the plane contains an uncountable collection of pairwise disjoint copies of $X$ ?

Question 2 (Problem 134 of [1]; see also Problem 3.8 of [1]). Is there a tree-like continuum $X$ with positive span which has the property that the plane contains uncountably many pairwise disjoint homeomorphic copies of $X$ ?

Question 3 (See Problem 9 of [12]). Is there a hereditarily indecomposable nonchainable continuum with span zero?

Regarding Question 1, we remark that the continua in Ingram's family from [6] are hereditarily indecomposable but not mutually homeomorphic.

\section{REFERENCES}

[1] Howard Cook, W. T. Ingram, and Andrew Lelek, A list of problems known as Houston problem book, Continua (Cincinnati, OH, 1994), Lecture Notes in Pure and Appl. Math., vol. 170, Dekker, New York, 1995, pp. 365-398. MR1326857 (96f:54042)

[2] Edward G. Effros, Transformation groups and $C^{*}$-algebras, Ann. of Math. (2) 81 (1965), 38-55. MR0174987 (30:5175)

[3] Charles L. Hagopian, Indecomposable homogeneous plane continua are hereditarily indecomposable, Trans. Amer. Math. Soc. 224 (1976), no. 2, 339-350. MR0420572 (54:8586)

[4] L. C. Hoehn, A non-chainable plane continuum with span zero, Fund. Math. 211 (2011), no. 2, 149-174. MR2747040 (2012d:54035)

[5] W. T. Ingram, An uncountable collection of mutually exclusive planar atriodic tree-like continua with positive span, Fund. Math. 85 (1974), no. 1, 73-78. MR0355999 (50:8472)

[6] _ Hereditarily indecomposable tree-like continua, Fund. Math. 103 (1979), no. 1, 6164. MR535836 (80i:54044)

[7] F. Burton Jones, On a certain type of homogeneous plane continuum, Proc. Amer. Math. Soc. 6 (1955), 735-740. MR0071761 (17:180e)

[8] B. Knaster and C. Kuratowski, Problème 2, Fund. Math. 1 (1920). 
[9] R. L. Moore, Concerning triods in the plane and the junction points of plane continua, Proc. Natl. Acad. Sci. USA 14 (1928), no. 1, 85-88.

[10] Sam B. Nadler, Jr., Continuum theory: an introduction, Monographs and Textbooks in Pure and Applied Mathematics, vol. 158, Marcel Dekker Inc., New York, 1992. MR.1192552 (93m:54002)

[11] Lex G. Oversteegen and E. D. Tymchatyn, Plane strips and the span of continua. I, Houston J. Math. 8 (1982), no. 1, 129-142. MR666153(84h:54030)

[12] (85h:54068)

[13] S. Todorčević, Embeddability of $K \times C$ into X, Bull. Cl. Sci. Math. Nat. Sci. Math. 22 (1997), 27-35. MR1612441 (98k:54023)

[14] Gerald S. Ungar, On all kinds of homogeneous spaces, Trans. Amer. Math. Soc. 212 (1975), 393-400. MR0385825 (52:6684)

[15] Eric K. van Douwen, Uncountably many pairwise disjoint copies of one metrizable compactum in another, Topology Appl. 51 (1993), no. 2, 87-91. MR.1229705 (94f:54034)

Department of Mathematics, University of Alabama at Birmingham, Birmingham, Alabama 35294-1170

E-mail address: 1hoehn@uab.edu

Current address: Department of Computer Science and Mathematics, Nipissing University, 100 College Drive, Box 5002, North Bay, Ontario, Canada P1B 8L7

E-mail address: loganh@nipissingu.ca 